\title{
An intelligent FPGA based anti-sweating system for bed sore prevention in a clinical environment
}

\author{
K.S. Jaichandar ${ }^{\mathrm{a}, *}$, Sampath Kumar ${ }^{\mathrm{a}}$, Chew kweeTiang ${ }^{\mathrm{b}}$, Siew Fong ${ }^{\mathrm{b}}$ and Stewart Tai ${ }^{\mathrm{b}}$ \\ ${ }^{a}$ Bio Medical Electronics Lab, School of Electrical and Electronics Engineering, Singapore Polytechnic, \\ Singapore \\ b Alexandra Hospital, Singapore
}

\begin{abstract}
Bed sores, a common problem among immobile patients occur as a result of continuous sweating due to increase in skin to bed surface temperature in patients lying on same posture for prolonged period. If left untreated, the skin can break open and become infected. Currently adopted methods for bed sores prevention include: use of two hourly flip chat for repositioning patient or use of air fluidized beds. However, the setbacks of these preventive measures include either use of costly equipment or wastage of human resources. This paper introduces an intelligent low cost FPGA based anti-sweating system for bed sores prevention in a clinical environment. The developed system consists of bed surface implanted temperature sensors interfaced with an FPGA chip for sensing the temperature change in patient's skin to bed surface. Based on the temperature change, the FPGA chip select the - mode (heater/cooler) and speed of the fan module. Furthermore, an alarm module was implemented to alert the nurse to reposition the patient only if patient's skin to bed surface temperature exceeds a predefined threshold thereby saving human resources. By integrating the whole system into a single FPGA chip, we were able to build a low cost compact system without sacrificing processing power and flexibility.
\end{abstract}

Keywords: Bed sores, skin to bed surface temperature, clinical environment, immobile patients

\section{Introduction}

Statistics have shown that about one million people in the United States suffer from bed sores ranging from mild inflammation to deep wounds, a research done by the Federal Agency reported that bed sores afflict: $10 \%$ of hospital patients, $25 \%$ of nursing home residents and $60 \%$ of quadriplegics [1]. The problem is also growing worse in developed countries partly because of an increasing aging population who when admitted

\footnotetext{
*Corresponding author: K.S. Jaichandar, Bio Medical Electronics Lab, School of Electrical and Electronics Engineering, Singapore, Polytechnic, 500 Dover Road, 139651 Singapore. Tel.: 0065 68790628; E-mail: jai@sp.edu.sg.
}

to hospitals are bedridden for months or even years due to the nature of the illnesses. Bedridden patients who are paralyzed or recovering from surgery are more prone to develop bedsore. Bed sores if left untreated causes the skin to break open and become infected. In more serious cases, bed sores causes damages ranging from a change in the color of unbroken skin to as severe as deep wounds, which can go to the muscle or bone. Prevention, rather than treatment, appears to be essential to reducing the burden of suffering associated with bed sore. Currently adopted methods for bed sores prevention include: use of two hourly flip chat for repositioning patient or use of air fluidized beds [2, $3]$. Currently various research groups are developing 


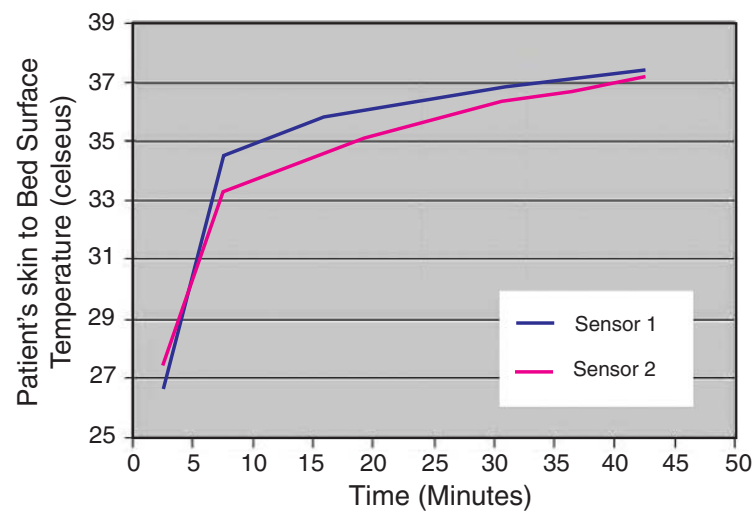

Fig. 1. Patient's skin to bed surface temperature versus time over which patient lies on the same posture.

solutions for the bed sore problem. Observations indicate that use of low-air-loss mattress a mechanical system reduces the size and facilitates the healing of bed sores [4]. A PC controlled automated system to flip the patient every 2 hours to minimise the bed sore has been developed [5]. A microcontroller based semi autonomous system to control the bed sore prevention was introduced [6]. How ever the current approaches are either expensive or impractical in clinical environment. The drawbacks of these preventive measures include wastage of human resources or use of costly equipments.

Bed sores occur as a result of increase in skin to bed surface temperature in patient lying on the same posture for prolonged period [7]. Figure 1 depicts the result of the experiments conducted with temperature sensors implanted on the bed surface, from the figure it is evident that as the time over which a patient lies in the same posture on the bed increases the patient's skin to bed surface temperature increases thereby increasing the chances of occurrence of bed sores in patient.
This paper introduces an intelligent low cost FPGA based anti-sweating system for bed sores prevention in a clinical environment. By integrating the whole system into a single FPGA chip, we were able to build a low cost compact system without sacrificing processing power and flexibility.

\section{Hardware description}

The implemented system shown in Fig. 2 consists of six modules namely, sensor module, Bio amplifier module, control module, alarm module, stepper motor and dimmer module and fan module.

\subsection{Sensor module}

In our experiment, four arrays of LM35 temperature sensors (two in each array) were used for sensing the patient's skin to bed surface temperature [5]. The LM35 sensor produces output voltage which is linearly proportional to the temperature. The output voltages of each of the four LM 35 sensors were calibrated for different temperature ranges using a black body [6] such that each $1.0 \mathrm{~V}$ output from the sensor corresponding to $10^{\circ}$ Celsius. A specialized mattress was fabricated with the temperature sensors implanted on them. Experiments were performed to determine potential spots on mattress to implant the temperature sensors. Figure 3 shows the test bed and fabricated mattress with temperature sensors implanted on potential spots.

\subsection{Bio amplifier module}

This module consists of two LF353 amplifiers, with internally trimmed input offset voltage to amplify

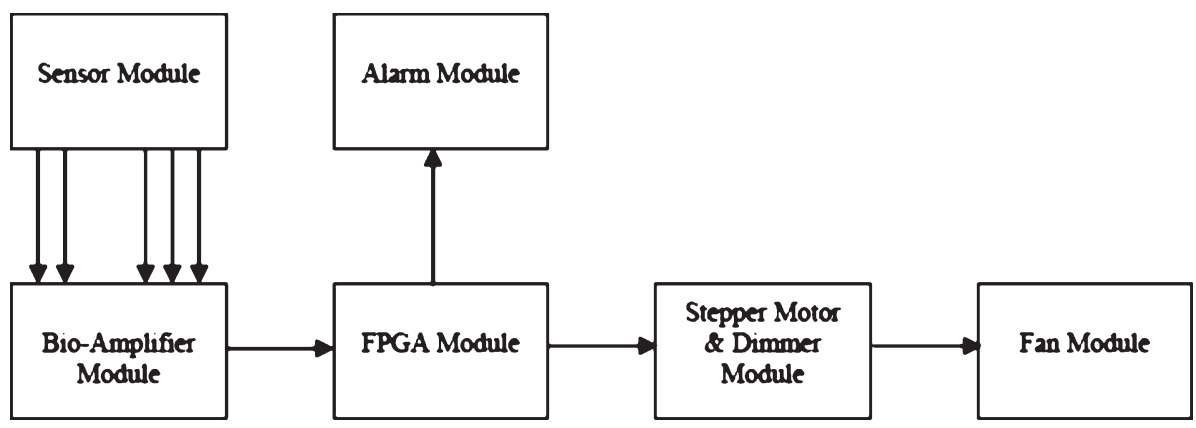

Fig. 2. Block diagram of the implemented system. 


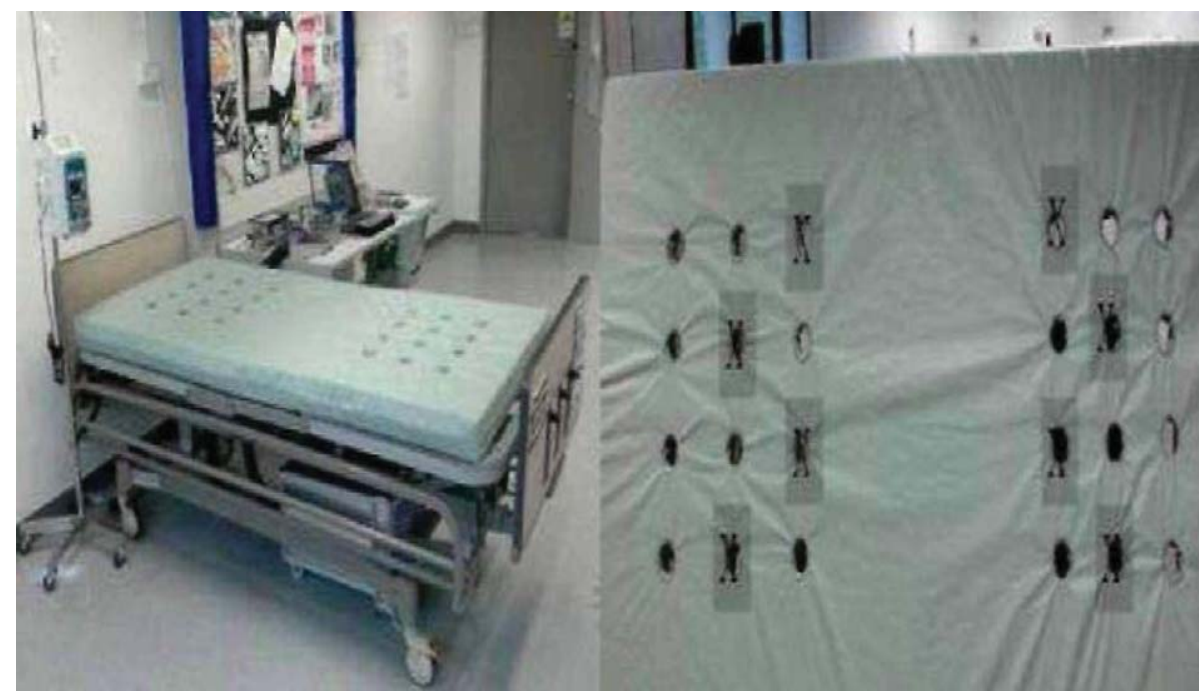

Fig. 3. Fabricated mattress with temperature sensors (Marked with X) implanted on potential spots.

the voltage signal from the linear temperature sensors implanted on the test bed [7]. For easiness in terms of computation an amplification factor of 10 was chosen.

\subsection{Field programmable gate array (FPGA) module}

Reconfigurable hardware is gaining popularity as compared to both Application Specific Integrated Circuits (ASIC). ASIC and general purpose processors for embedded applications. Field Programmable Gate Array (FPGA) based systems have been adopted in applications ranging from robotics to bio medical signal processing. Field Programmable Gate Arrays (FPGAs) have better flexibility and shorter design cycle than Application Specific Integrated Circuits (ASIC). Due to ever-increasing integrated circuit fabrication capabilities, the future of FPGA technology promises both higher densities and higher speeds.

Design entry was achieved using a system level design language, Handle C. It is a high level FPGA implementation language with an ANSI C syntax and some hardware related language features such as parallel execution, channel communication, interface definition, etc. Handel-C is more convenient for rapid prototyping of control-centric algorithms as compared to other hardware description languages such as VHDL or Verilog HDL [8].
The FPGA hardware used to for our implementation is a RC100 development board from Celoxica with an inbuilt Xilinx Spartan-II FPGA. The Spartan-II device on the RC100 board is a XC2S200 with 2,00,000 gates and 14 blocks of $4 \mathrm{Kbits}$ RAM. The main control tasks handled by the FPGA module are to,

Digitalize the analog voltage signals from the Bio Amplifier.

- Control a stepper motor which controls the fan module.

- Control an alarm module (consisting of a beeper and LED display).

\subsection{Stepper motor and dimmer module}

A dimmer mounted on a stepper motor shown in Fig. 4 was used for controlling the fan speed. This module makes the whole system automated as the control of fan speed was achieved without any human intervention.

\subsection{Fan module}

A specialized fan module shown in Fig. 5 consisting of a heater and a cooler was fabricated. The heater was integrated to the fan module so as to soothe the patient by blowing warm air to the bed surface if the patient's skin to bed surface temperature falls below a predefined temperature value. Depending on the patient's skin to 


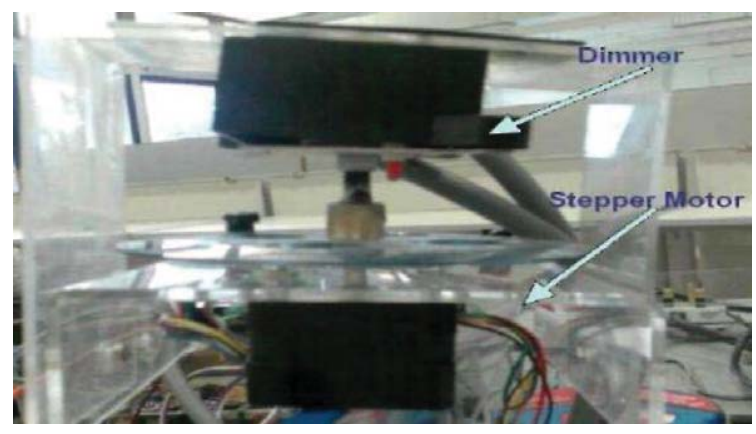

Fig. 4. Dimmer mounted on the stepper motor for fan speed control.

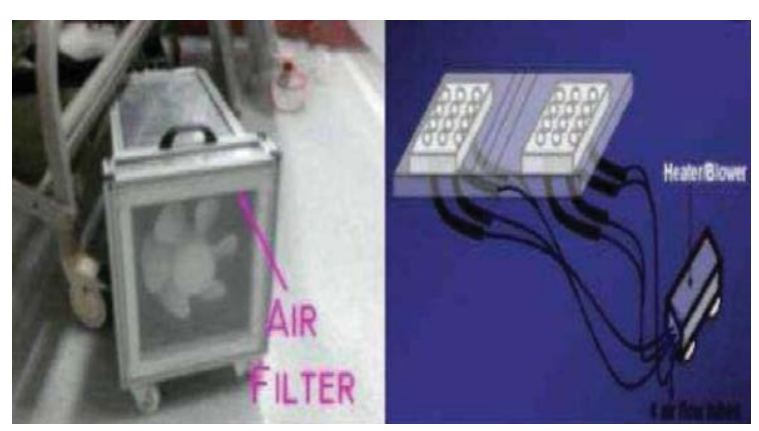

Fig. 5. Fan module

bed surface temperature either the heater or the cooler integrated to the fan will be turned ON. Four different fan speeds were chosen for our experiments. A filter was implemented together with the fan unit to filter out dust particles.

\subsection{Software description}

The main aspect of the FPGA programmed software is to monitor and control the patient's skin to bed surface temperature in order to reduce the occurrences of bed sores.

\subsubsection{Algorithm}

The logical flow of the developed algorithm begins with acquisition of amplified temperature equivalent voltage signal from all the temperature sensors. Algorithm compares the acquired sensor signals and only selects the largest temperature equivalent voltage signal. The chosen voltage signal is then categorized into one of the predefined ranges depending on its value. If the chosen voltage signal is less than or equal to
$2.5 \mathrm{~V}\left(25^{\circ} \mathrm{C}\right)$, then the algorithm activates the heater and drives the fan at speed 1 . If the chosen voltage signal is greater than $2.5 \mathrm{~V}\left(25^{\circ} \mathrm{C}\right)$ then the algorithm turns OFF the heater. For voltage signals between $2.5 \mathrm{~V}$ and $3.1 \mathrm{~V}\left(25^{\circ} \mathrm{C}\right.$ and $\left.31^{\circ} \mathrm{C}\right)$, the algorithm activates the cooler and drives the fan at speed 1 . For voltage signals between $3.1 \mathrm{~V}$ and $3.8 \mathrm{~V}\left(31^{\circ} \mathrm{C}\right.$ and $\left.38^{\circ} \mathrm{C}\right)$, the algorithm activates the cooler and drives the fan at speed 2. If the voltage is neither one, the algorithm activates the cooler, drives the fan at speed 3 and also activates the alarm consisting of a beeper and LED display alerting the nurse in charge to check and reposition the patient. The developed system has an ON/OFF switch enabling the user to activate/deactivate the system at any point of time. Fan speed 1 corresponds slow speed and 3 corresponds to faster and 2 is in-between 1 and 3 .

\section{Experiments and results}

Mock experiments were performed with 15 patients for duration of 2 hours. Selected patients were allotted serial numbers and were asked to wear a cotton shirt. The room temperature in the lab were experiments were conducted was recorded to be $30.4^{\circ} \mathrm{C}$ with a humidity of $76 \%$. The weights of the cotton shirt provided to the patients were recorded as shown in Table 1 as WB1 in gm. Patients lied on the normal hospital bed for 2 hours and then the weights of their cotton shirts were measured and recorded as WN (in gm) as shown in Table 1. The amount of sweat for each patient lying for 2 hours with normal hospital bed was calculated.

The values for the amount of sweat for each patient lying for 2 hours with normal hospital bed are tabulated in Table 1. The experiment was repeated for FPGA based intelligent bed system. The amount of sweat for each patient lying for 2 hours with FPGA based intelligent bed system was calculated and tabulated in Table 1. From Table 1, it is very obvious on comparison that the amount of sweat for patients can be greatly reduced with our FPGA based intelligent bed system. Also from the table the average Temperature reinforces that the special bed offers better bed sore prevention capabilities as compared with normal bed.

Another experiment followed by a survey was performed with a group 30 people and $96.67 \%$ of them felt that the system developed would be efficient and effective in preventing bed sores. 
Table 1

Experiment result tabulation

\begin{tabular}{|c|c|c|c|c|c|c|}
\hline $\begin{array}{l}\text { Patient } \\
\text { number }\end{array}$ & $\begin{array}{l}\text { Weight of cotton } \\
\text { shirt before } \\
\text { experiment, } \\
\text { W }_{\text {B1 (in gms) }}\end{array}$ & $\begin{array}{l}\text { Weight of cotton } \\
\text { shirt after lying } \\
\text { on the normal bed } \\
\text { for } 2 \text { hours, } \\
\mathrm{W}_{\mathrm{N}} \text { (in gms) }\end{array}$ & $\begin{array}{l}\text { Amount of sweat } \\
\text { with normal bed, } \\
\mathrm{W}_{\mathrm{S} 1}=\mathrm{W}_{\mathrm{N}}-\mathrm{W}_{\mathrm{B} 1} \\
\quad \text { (in gms) }\end{array}$ & $\begin{array}{l}\text { Weight of cotton } \\
\text { shirt before } \\
\text { experiment, } \\
\text { WB2 (in gms) }\end{array}$ & $\begin{array}{l}\text { Weight of cotton } \\
\text { shirt after lying } \\
\text { on the specialized } \\
\text { bed for } 2 \text { hours, } \\
\text { W }_{\text {I (in gms) }}\end{array}$ & $\begin{array}{c}\text { Amount of sweat } \\
\text { with specialized bed, } \\
\mathrm{W}_{\mathrm{S} 2}=\mathrm{W}_{\mathrm{I}}-\mathrm{W}_{\mathrm{B} 2} \\
\text { (in gms) }\end{array}$ \\
\hline 1 & 402 & 457 & 55 & 398 & 417 & 19 \\
\hline 2 & 406 & 465 & 59 & 402 & 419 & 17 \\
\hline 3 & 401 & 465 & 64 & 400 & 420 & 20 \\
\hline 4 & 399 & 452 & 53 & 402 & 413 & 11 \\
\hline 5 & 403 & 463 & 60 & 399 & 415 & 16 \\
\hline 6 & 398 & 441 & 43 & 403 & 407 & 4 \\
\hline 7 & 404 & 466 & 62 & 402 & 412 & 10 \\
\hline 8 & 401 & 458 & 57 & 399 & 416 & 17 \\
\hline 9 & 400 & 461 & 61 & 401 & 414 & 13 \\
\hline 10 & 398 & 443 & 45 & 403 & 409 & 6 \\
\hline 11 & 397 & 452 & 55 & 400 & 411 & 11 \\
\hline 12 & 400 & 459 & 59 & 398 & 417 & 19 \\
\hline 13 & 402 & 456 & 54 & 397 & 413 & 16 \\
\hline 14 & 398 & 449 & 51 & 401 & 412 & 11 \\
\hline 15 & 399 & 462 & 63 & 406 & 421 & 15 \\
\hline
\end{tabular}

\section{Conclusion}

The control and monitoring of patient's skin to bed surface temperature is one of the vital aspects for the prevention of bed sore. Providing air ventilation to the bed surface not only aids in the prevention of bed sore but also provides comfort to the patient.

The main focus of this paper is on the development of an intelligent low cost FPGA based anti-sweating system for bed sores prevention in a clinical environment. Experiments were performed to study the usefulness of the developed system and also a comparative study was made against the conventional hospital bed.

In this paper, we have also discussed about the hardware and software descriptions of our system. With gaining popularity for reconfigurable FPGA hardware, the system was implemented using a RC100 development board from Celoxica with an inbuilt Xilinx Spartan-II FPGA to make decisions on the mode (heater/cooler) and speed of the fan depending on the inputs from the temperature sensor. The FPGA module also alerts the nurse in charge to reposition the patient when his/her skin to bed surface temperature exceeds a predefined threshold. The effectiveness of the proposed method was verified with repeated experiments and satisfactory results were obtained.

\section{References}

[1] https://www.healthatoz.com/healthatoz/Atoz/common/stand ard/transform.jsp?requestURI=/healthatoz/Atoz/ency/bed sores.jsp.

[2] C. Sharp, G. Burr, M. Broadbent, M. Cummins, H. Casey and A. Merriman, Pressure ulcer prevention and care: A survey of current practice, J Qual Clin Pract 20(4) (2000), 150-157.

[3] M. Allman, J.M. Walker, M.K. Hart, C.A. Laprade, L.B. Noel and C.R. Smith, Air-fluidized beds or conventional therapy for pressure sores: A randomized trial, Annals of internal medicine 107(5) (1987), 641-648.

[4] M.A. Charles, J. Oldenbrook and C. Catton, Evaluation of a low-air-loss mattress system in the treatment of patients with pressure ulcers, Ostomy Wound Manage 4(5) (1995), 46-52.

[5] S. Ahmed, T. Shimada, A. Funakubo, T. Dohi and Y. Fukui, Development of a bedsore preventive mattress for physically Disabled person, IFMBE Proceedings, 14 (2007), 2939-2940.

[6] K.S. Jaichandar, Mohan Rajesh Elara, Sampath Kumar and Chua Adrian, A semi-Autonomous control and monitoring system for Bed Sore Prevention, ICREAT (2007), 245-248.

[7] A.C. Ek, G. Gustavsson and D.H. Lewis, Skin blood flow in relation to external pressure and temperature in the supine position on a standard hospital mattress, Scand J Rehabil Med 19 (1987), 121-126

[8] K.V. Ling, S.P. Yue and J.M.A. Maciejowski, FPGA Implementation of Model Predictive Control. ACC'06 June. 

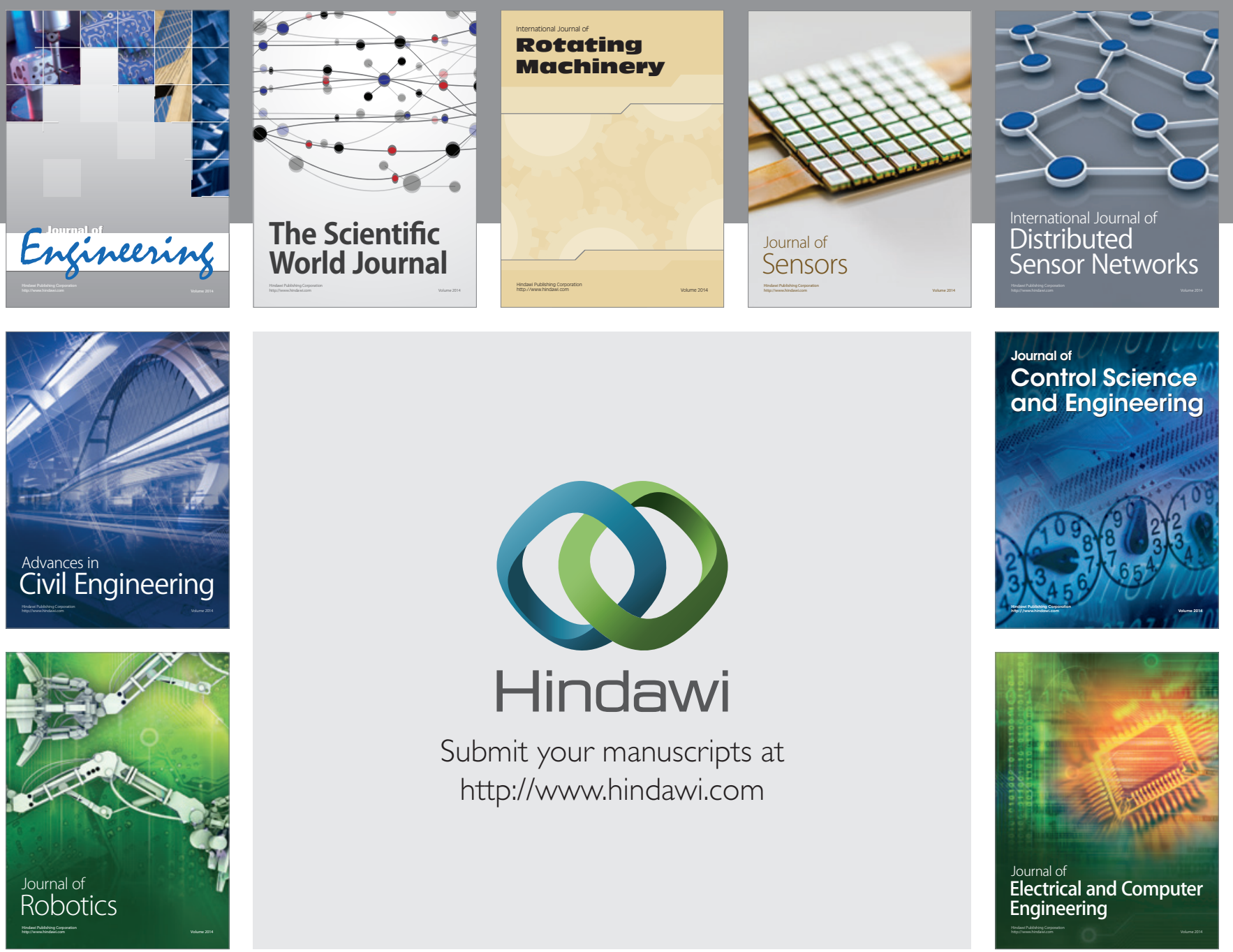

Submit your manuscripts at

http://www.hindawi.com
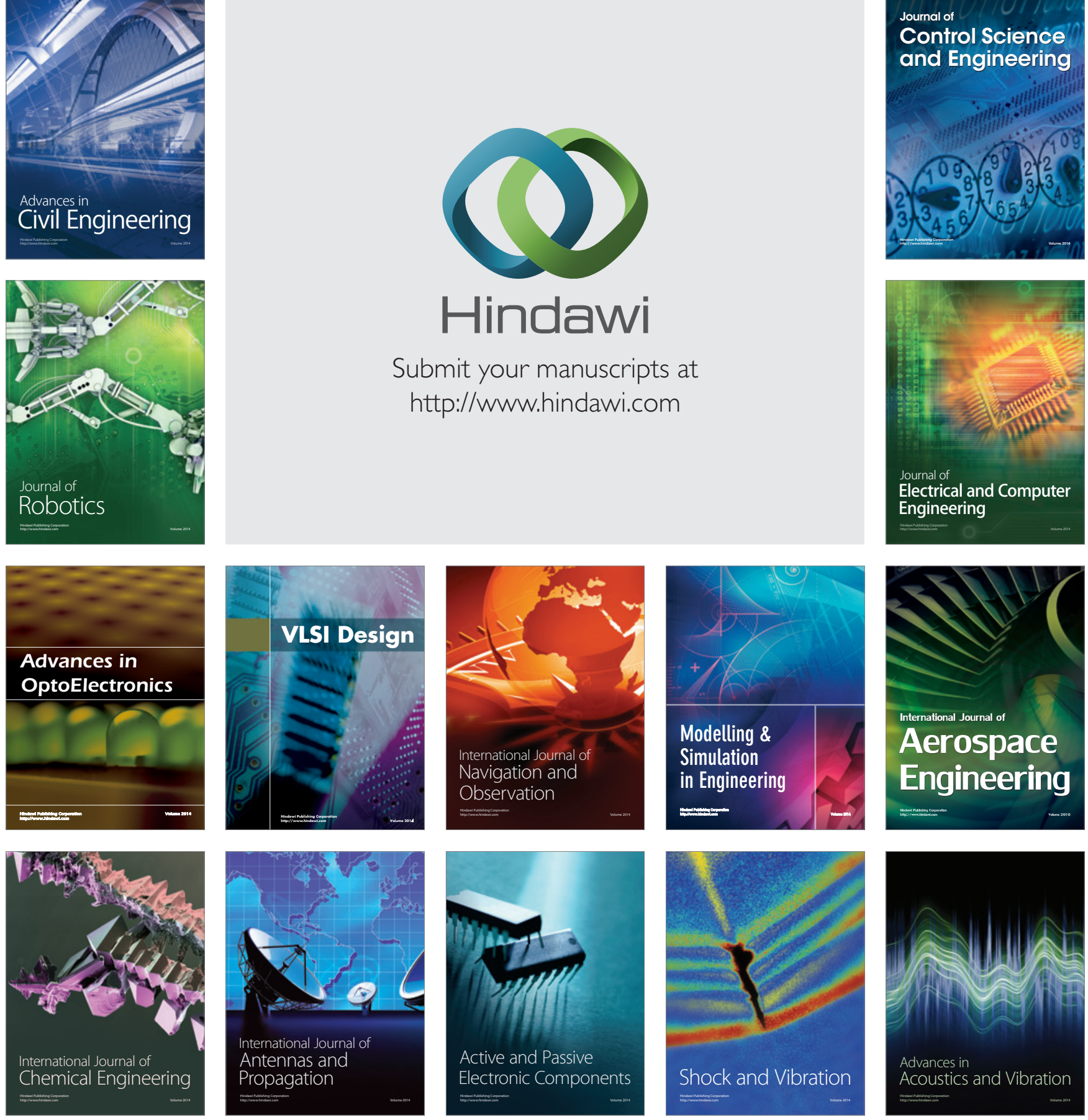